\title{
Transmembrane routes of cationic liposome-mediated gene delivery using human throat epidermis cancer cells
}

\author{
Shaohui Cui • Bing Wang • Yinan Zhao • \\ Huiying Chen · Huiqin Ding • Defu Zhi • \\ Shubiao Zhang
}

Received: 5 December 2012/ Accepted: 8 August 2013/Published online: 26 September 2013

(C) The Author(s) 2013. This article is published with open access at Springerlink.com

\begin{abstract}
For studying the mechanism of cationic liposome-mediated transmembrane routes for gene delivery, various inhibitors of endocytosis were used to treat human throat epidermis cancer cells, Hep-2, before transfection with Lipofectamine 2000/pGFP-N2 or Lipofectamine 2000/pGL3. To eliminate the effect of inhibitor toxicity on transfection, the RLU/survival rate was used to represent the transfection efficiency. Chlorpromazine and wortmannin, clathrin inhibitors, decreased transfection efficiency by $44 \%(100 \mu \mathrm{M})$ and $31 \%(100 \mathrm{nM})$, respectively. At the same time, genistein, a caveolin inhibitor, decreased it by $30 \%$ $(200 \mu \mathrm{M})$. Thus combined transmembrane routes through the clathrin and caveolae-mediated pathways were major mechanisms of cell uptake for the cationic liposome-mediated gene delivery. After entering the cells, microtubules played an important role on gene delivery as vinblastine, a microtubulin inhibitor, could reduce transfection efficiency by $41 \%(200 \mathrm{nM})$.
\end{abstract}

Keywords Cancer cells - Cationic liposome · Endocytic pathway $\cdot$ Gene delivery $\cdot$ Human throat cancer cells · Inhibitor - Transfection efficiency

S. Cui $\cdot$ B. Wang $\cdot$ Y. Zhao $\cdot$ H. Chen

H. Ding $\cdot$ D. Zhi $\cdot$ S. Zhang $(\bowtie)$

SEAC-ME Key Laboratory of Biotechnology and Bioresouces Utilization, College of Life Science, Dalian Nationalities University, Dalian 116600, Liaoning, China e-mail: 2356166886@qq.com

\section{Introduction}

Although non-viral vectors are inferior compared to viral vectors in terms of transfection efficiency, they have the advantages over viral ones as they are nonimmunogenic, easy to produce and are not oncogenic. Among non-viral vectors, cationic liposomes are used extensively as gene carriers for plasmid DNA (pDNA), antisense oligonucleotides and small interfering RNA (High 2005; Obata et al. 2010). Because the cell membrane is the first barrier for liposome/ DNA complexes (lipoplexes) to enter into cells, the mechanism of cationic liposome-mediated transmembrane gene delivery is important as a study of it could contribute to improving transfection efficiency.

Endocytosis is generally considered to be the main entering pathway for lipoplexes (Chittimalla et al. 2005; Payne et al. 2007). There are however, various endocytic pathways in eukaryotic cells, such as clathrin-dependent and clathrin-independent pathways. The latter includes phagocytosis, macropinocytosis and caveolae-mediated internalization (Sandvig et al. 2011). The relative contribution of each pathway in lipoplexes internalization has been poorly defined to date (Mayor and Pagano 2007; Howes et al. 2010). Endocytotic inhibitors, such as chloroquine, chlorpromazine, $\mathrm{NaN}_{3}$ or filipin, are helpful for studying drug intracellular release or elucidating a specific endocytosis route (Blanchard et al. 2006). There are still many problems that have not been elucidated on the biological barriers although much effort has been 
expended on them. As non-viral vectors are less efficient than the viral ones, therefore understanding the mechanism of transmembrane might contribute to overcoming the hurdles in gene delivery. This study was aimed to elucidate the transmembrane pathways of gene delivery mediated by a cationic liposome, Lipofectamine 2000, while the inhibitors such as chlorpromazine (wortmannin), genistein and vinblastine for inhibiting clathrin, caveolin and microtubulin pathways, respectively, were used to treat Hep-2 cells before transfection.

\section{Materials and methods}

\section{Materials and reagents}

Human throat epidermis (Hep-2) cancer cell line was purchased from Cell Bank of Chinese Academy of Sciences. Lipofectamine 2000 was from Invitrogen. The pGL3-control (pLuc) vector plasmid (Promega) containing a modified coding region for firefly (Photinus pyralis) luciferase and the pGFP-N2 vector plasmid (Clontech) containing a coding region for green fluorescence protein were used for transfection. Bright-Glo Luciferase Assay System was purchased from Promega.

Cell culture

Hep-2 cells were grown in a $100 \mathrm{ml}$ culture flask in RPMI-1640 medium supplemented with $10 \%$ (v/v) FBS and antibiotics (penicillin $100 \mathrm{U} / \mathrm{ml}$ and streptomycin $100 \mathrm{U} / \mathrm{ml}$ ) at $37{ }^{\circ} \mathrm{C}$ in a humidified atmosphere containing $5 \%(\mathrm{v} / \mathrm{v}) \mathrm{CO}_{2}$.

Plasmid preparation

pGL3-control and pGFP-N2 plasmids were amplified in Escherichia coli (strain JM109 and DH5-a, respectively) and purified using an endotoxin-free kit (Qiagen) to remove the bacterial endotoxins. The quantity and quality of purified pDNA were assessed at 260 and $280 \mathrm{~nm}$ by electrophoresis in agarose gels.

\section{Lipoplexes preparation}

For the preparation of lipoplexes, $0.3 \mu \mathrm{l}$ pDNA (pGFP$\mathrm{N} 2$ or pGL3) $(1 \mathrm{mg} / \mathrm{ml})$ and $0.6 \mu 1$ Lipofectamine 2000 $(1 \mathrm{mg} / \mathrm{ml})$, were diluted in Opti-MEM medium $25 \mu \mathrm{l}$, respectively, and then the diluted transfection reagent was added to pDNA solutions. The mixtures were vortexed gently and incubated for $20 \mathrm{~min}$ at room temperature for 96 well plate transfection.

Transfection procedure

Hep-2 cells $\left(10^{5}\right.$ cells $\left./ \mathrm{ml}\right)$ were seeded into 96-well plates in $100 \mu$ l growth medium (RPMI-1640) until the required cell number was obtained (80\% confluence) at the time of transfection. Cells were washed once with PBS, and then $50 \mu 1$ lipoplexes (prepared as described above) were added to each well. It was mixed gently by rocking the plate. The cells were incubated for 4-6 h, washed by PBS once and the growth medium was replaced. Cells were further cultured for $48 \mathrm{~h}$ prior to analysis.

Luciferase assay

Relative luciferase activity was assessed using the Bright-Glo Luciferase Assay System and a microplate reader. The growth medium was removed from each well, cells were rinsed once with PBS and luciferase activity was measured after $100 \mu$ lysis buffer was added into each well of the 96-well plate with the incubation of $5 \mathrm{~min}$ at room temperature. The protein concentrations of cell lysates per well were determined using the BCA protein assay kit, and then the transfection efficiency was obtained as the relative luciferase activity. Data were expressed as relative light units (RLU) per mg protein. Each data point was averaged over three replicates.

Green fluorescence protein (GFP) assay

The expression of GFP was imaged by inverted fluorescence microscope and transfection efficiency was given as relative efficiency. The number of GFPexpressing cells versus the total cell quantity in the microscope was defined as the transfection efficiency. Cell counting was performed randomly in microscopic observation scope under $10 \times 20$ magnification with three repeats.

\section{Cytotoxicity assay}

The cytotoxicity was evaluated by MTT assay. Hep-2 cells $\left(10^{5}\right.$ cells $\left./ \mathrm{ml}\right)$ were seeded into 96 -well plates at 
$200 \mu \mathrm{l}$ per well and incubated $24 \mathrm{~h}$ before treatment. Various kinds of inhibitors were added to the cells and after $1 \mathrm{~h}, 50 \mu \mathrm{l}$ MTT ( $5 \mathrm{mg} / \mathrm{ml}$ in PBS) was added and incubated for an additional $4 \mathrm{~h}$. MTT-containing medium was removed and $150 \mu \mathrm{L}$ DMSO was added to dissolve the formazan and the absorbance was measured at $570 \mathrm{~nm}$ to determine cell survival as percentage of control. Data were presented as the mean \pm SD.

\section{Results}

The effects of clathrin inhibitors on transfection of lipoplexes

Endocytosis is the main process by which cells take up macromolecules. However, there are many types of endocytic pathways, among which the so-called clathrin-dependent endocytosis is well characterized. To determine the influence of clathrin-dependent endocytosis on cationic liposome-mediated transfection, two inhibitors (chlorpromazine and wortmannin) of clathrin were used to treat Hep-2 cells before transfection. The transfection efficiency of Lipofectamine
2000/pGFP-N2 complexes was investigated through the detection of GFP expression (Fig. 1). GFP expression obviously decreased with increasing inhibitor concentrations. When chlorpromazine was $100 \mu \mathrm{M}$, GFP expression was very low (Fig. 1a, b). In the same way, there was significant inhibition when wortmannin was higher than $60 \mathrm{nM}$ (Fig. 1c, d).

Additionally, luciferase reporter gene was used to examine the effects of inhibitors on transfection efficiency. Luciferase activity at $48 \mathrm{~h}$ after transfection was measured (Fig. 2): luciferase gene expression, as RLU in Hep-2 cells, was reduced by up to $66 \%$ when cells were treated with up to $100 \mu \mathrm{M}$ chlorpromazine (Fig. 2a) and by up $72 \%$ when cells were treated with up to $100 \mathrm{nM}$ wortmannin (Fig. 2c).

The cytotoxicity of chlorpromazine and wortmannin was tested by MTT assay, as these inhibitors could influence cell survival and evaluation of the transfection efficiency. Cell survival rates were between 87 and $60 \%$ when cells were treated cells chlorpromazine (Fig. 2a), and between 79 and $41 \%$ with wortmannin (Fig. 2c). To minimize the influence of toxicity of the inhibitors on transfection, the ratios of RLU to survival rate were used to show the inhibition of transfection. The RLU/survival rate also decreased
A
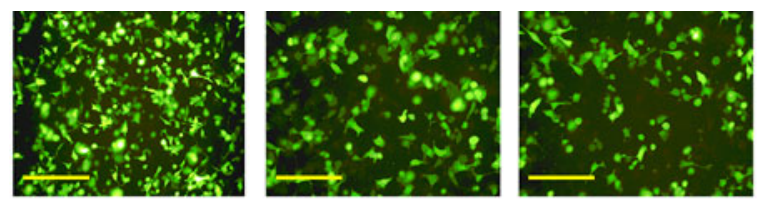

B
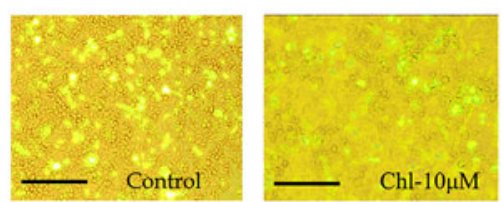

C
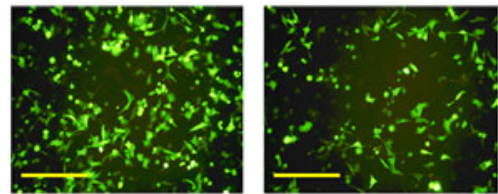

D
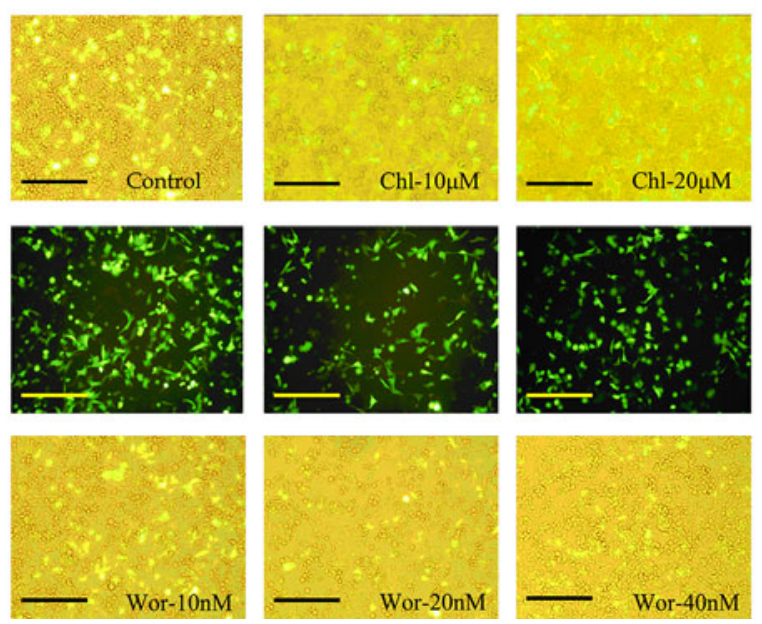

Fig. 1 Effect of concentrations of chlorpromazine $(10-100 \mu \mathrm{M})$ and wortmannin $(10-100 \mathrm{nM})$ on GFP expression mediated by Lipofecamine 2000/pGFP-N2 complexes against
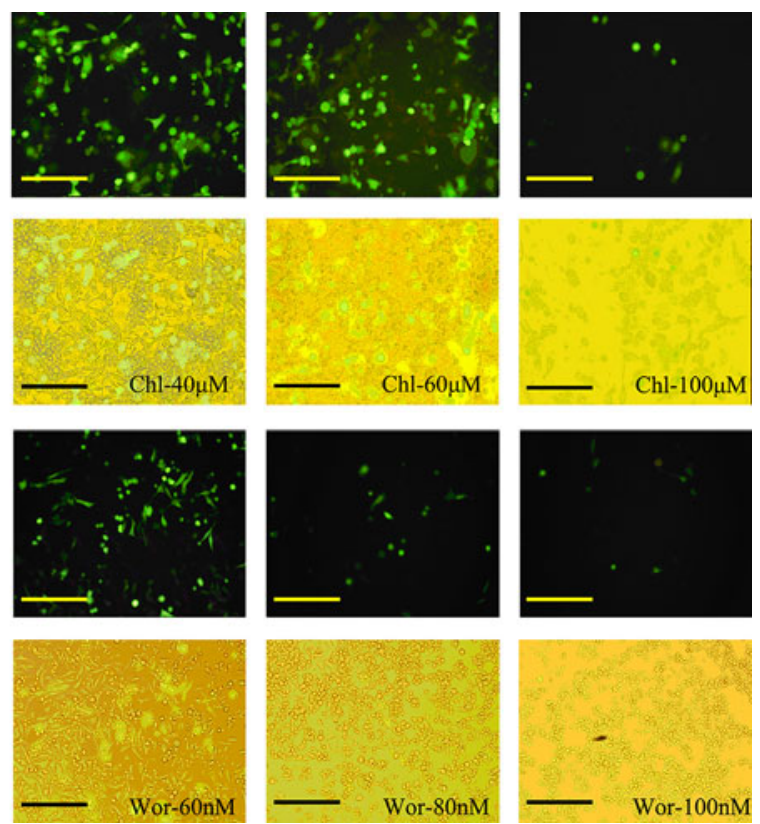

Hep- 2 cells. a, c The expression of GFP was imaged by inverted fluorescence microscope $(10 \times 20)$; $\mathbf{b}$, $\mathbf{d}$ contrast cells in bright field. Scale bar $=50 \mu \mathrm{m}$ 

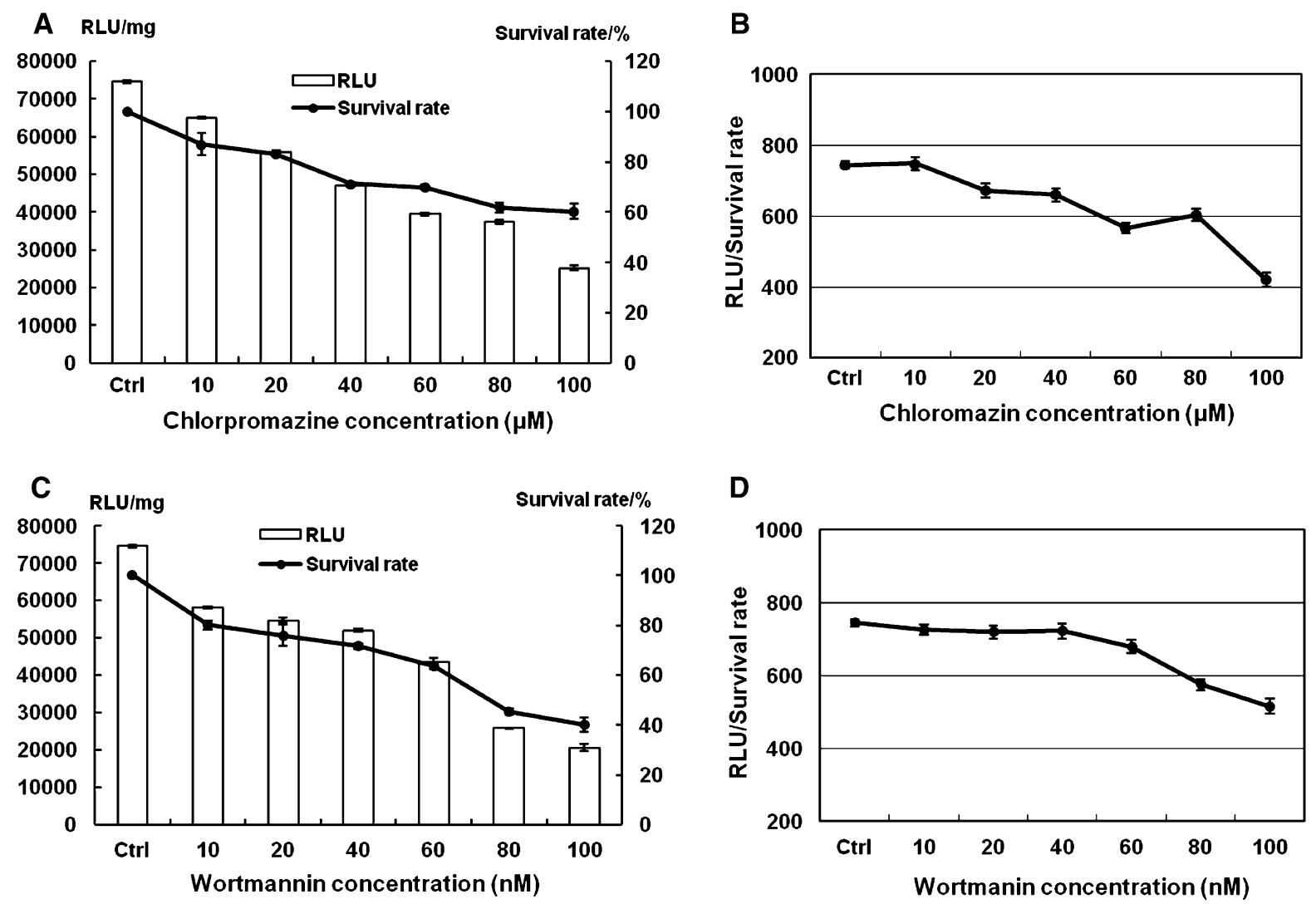

Fig. 2 Luciferase reporter gene expression and cell survival rate. a, c The evaluation of transfection efficiency and cell survival rate with chlorpromazine $(10-100 \mu \mathrm{M})$ and wortmannin (10-100 nM) treated Hep-2 cells. Lipofectamine 2000 was used as positive control in transfection. Cell survival rate was

with an increase of chlorpromazine and wortmannin concentrations. The expression of protein was reduced by up to $44 \%$ of the RLU/survival rate with chlorpromazine up to $100 \mu \mathrm{M}$ (Fig. 2b). For wortmannin, expression was reduced by up to $31 \%$ at $100 \mathrm{nM}$ (Fig. 2d). These results suggested that both chlorpromazine and wortmannin lower transfection efficiencies by inhibiting clathrin-dependent endocytosis.

The effects of caveolin inhibitor on transfection of lipoplexes

Besides clathrin-dependent endocytosis, another internalization pathway is caveolae-dependent. Genistein, the inhibitor of caveolin, which is essential for the formation and stability of caveolae, was used to treat Hep-2 cells $1 \mathrm{~h}$ before transfection. Fig. 3 shows that the transfection efficiency, in terms of GFP

determined with the MTT method with non-treated cells as control. All results are expressed as mean $\pm \operatorname{SD}(n=4)$. b, d RLU/survival rate with chlorpromazine $(10-100 \mu \mathrm{M})$ and wortmannin (10-100 nM) treated Hep-2 cells

expression, was reduced by up to $80 \%$ with up to $200 \mu \mathrm{M}$ genistein. Luciferase reporter gene expression showed that transfection efficiency was reduced by up to $62 \%$ with genistein up to $200 \mu \mathrm{M}$ compared to the control (Fig. 4a). The cytotoxicity of genistein to cells is shown in Fig. 4a. The ratios of RLU to survival rate showed the protein expression was decreased by $30 \%$ with $200 \mu \mathrm{M}$ genistein (Fig. 4b). These results demonstrated the route of transmembrane transport of lipoplexes by caveolae-dependent pathway also played an important role.

The effects of microtubule inhibitor on transfection of lipoplexes

Uptake of materials into cells and subsequent internalization is usually related to the function of microtubules. Some proteins are trafficked to the nucleus by 
A

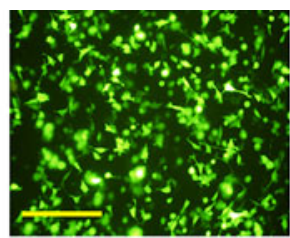

B

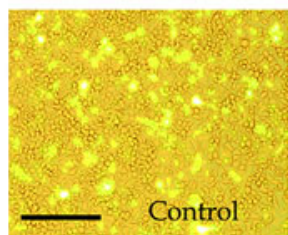

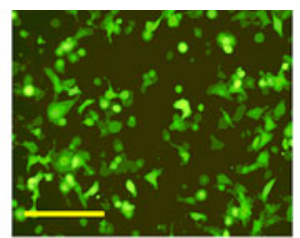

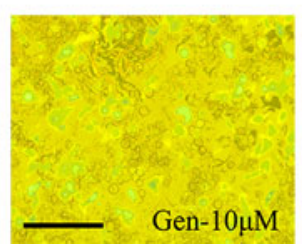

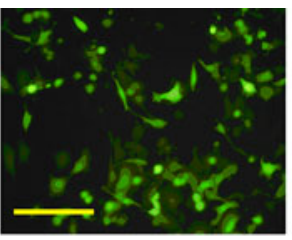

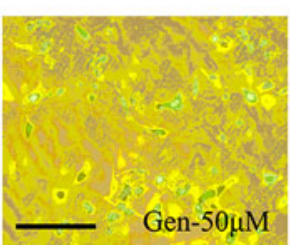

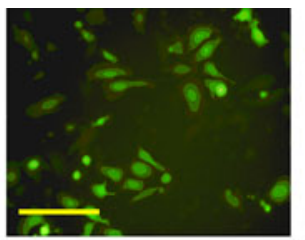
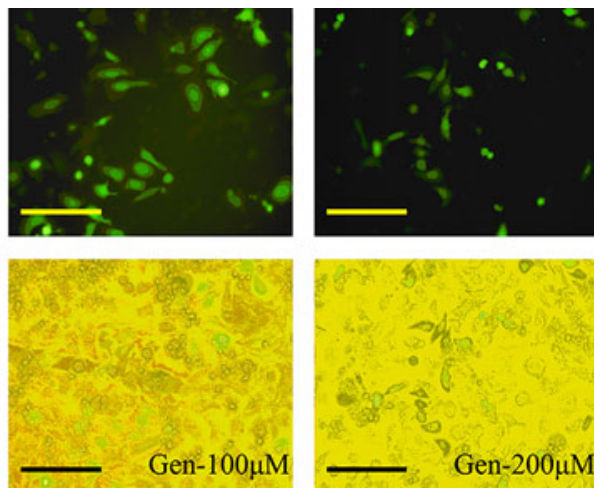

Fig. 3 Effect of concentrations of genistein (10-200 $\mu \mathrm{M})$ on GFP expression mediated by Lipofecamine 2000/pGFP-N2 complexes. a Inverted fluorescent microscope images $(10 \times 20)$; b contrast cells in bright field. Scale bar $=50 \mu \mathrm{m}$

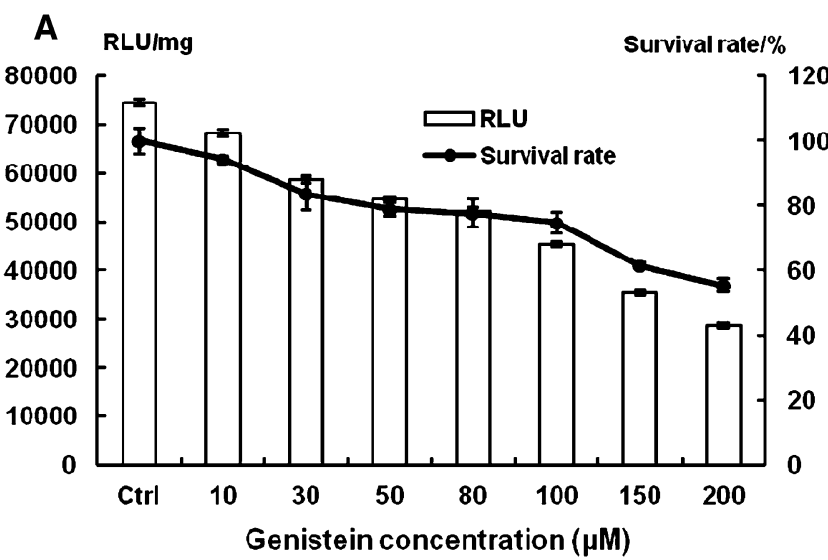

Fig. 4 Luciferase reporter gene expression and cell survival rate. a The evaluation of transfection efficiency and cell survival with genistein $(10-200 \mu \mathrm{M})$ treated Hep-2 cells. Lipofectamine 2000 was used as positive control. Cell survival rate was

microtubules and are further dismantled in cells. Vinblastine, which is an inhibitor of microtubule function, was used to treat cells $1 \mathrm{~h}$ before transfection. Then, in the same way, transfected cells were detected after the delivery of the reporter genes. The transfection efficiency of pGFP-N2 was reduced by up to $98.3 \%$ when cells were treated with up to $200 \mathrm{nM}$ vinblastine (Fig. 5). The results of luciferase reporter gene expression showed that transfection efficiency was reduced by up to $54 \%$ with vinblastine up to 200 $\mathrm{nM}$ (Fig. 6a). The cytotoxicity of vinblastine to cells is shown in Fig. 6a. RLU/survival rate (Fig. 6b) showed the expression of luciferase gene was reduced by up to $41 \%$ with vinblastine up to $200 \mathrm{nM}$. These results indicated that vinblastine could reduce the transfection
B

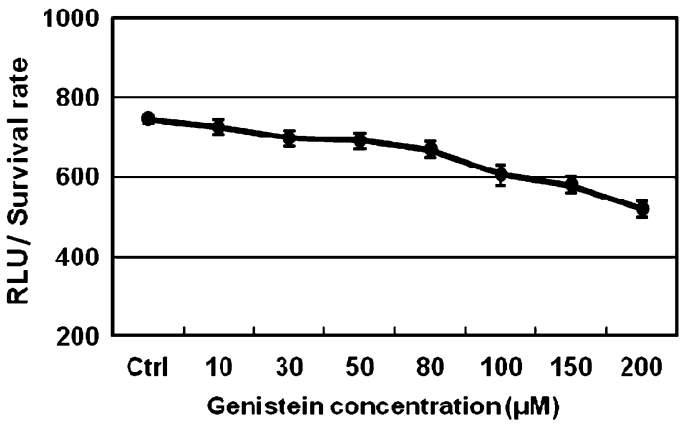

determined with the MTT method with non-treated cells as control. All results are expressed as mean $\pm \mathrm{SD}(n=4)$. b RLU/survival rate with genistein (10-200 $\mu \mathrm{M})$ treated Hep-2 cells

efficiency of lipoplexes through inhibiting the function of microtubulin.

\section{Discussion}

Knowledge about the transmembrane uptake mechanism is important for the development of efficient carriers for gene delivery. So far, some results indicate that endocytosis is the major mechanism of lipoplexes entry into cells. There are various endocytic pathways in eukaryotic cells, among them clathrin-mediated endocytosis functions by means of coated pits and endocytic internalization. Clathrin-independent endocytic pathways include phagocytosis, macropinocytosis and 
A

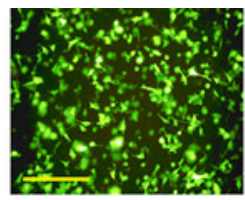

B

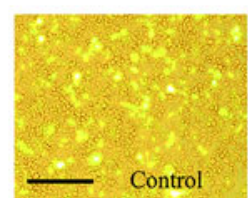

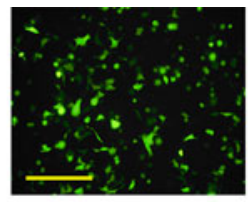

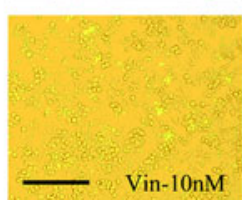

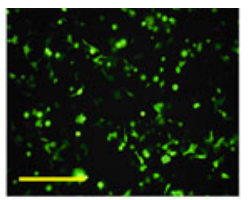
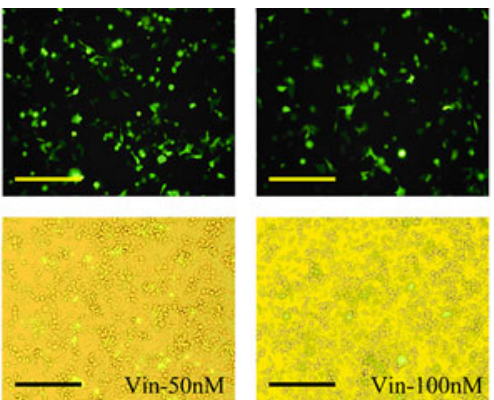
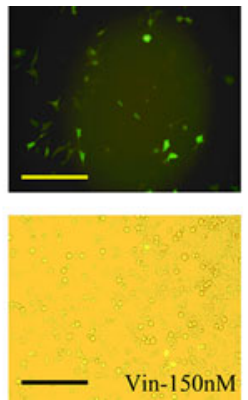

Fig. 5 Effect of concentrations of vinblastine (10-200 nM) on GFP expression mediated by Lipofecamine 2000/pGFP-N2 complexes. a Inverted fluorescent microscope images $(10 \times 20)$; b contrast cells in bright field. Scale bar $=50 \mu \mathrm{m}$

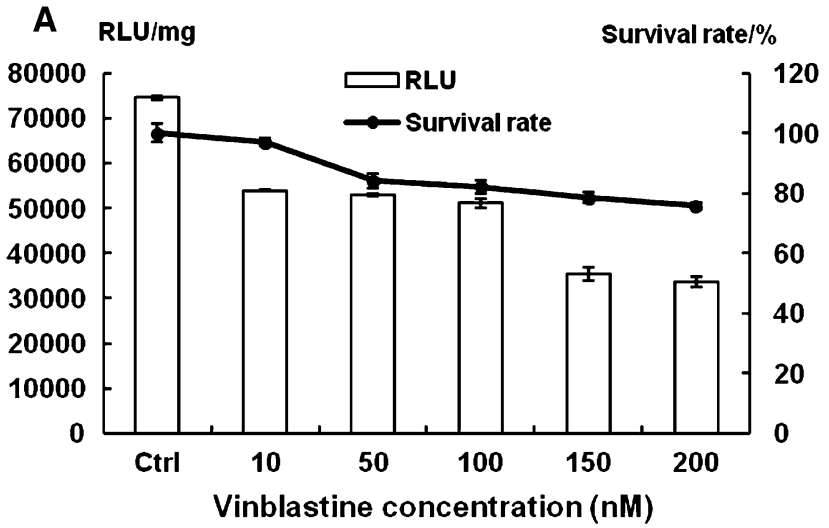

Fig. 6 Luciferase reporter gene expression and cell survival rate. a The evaluation of transfection efficiency and cell viability with vinblastine (10-200 nM) treated Hep-2 cells. Lipofectamine 2000 was used as positive control. Cell viability was

caveolae-mediated endocytosis (Payne et al. 2007; Ziello et al. 2010). But there are few studies to focus on the detailed transporting routes of cationic liposomemediated gene delivery. This study aimed to elucidate this problem by using different inhibitors to inhibit the pathways of cell uptake, hoping to provide reference for better understanding and overcoming the biological barriers which exist in the process of gene delivery.

von Gersdorff et al. (2006) showed that chlorpromazine inhibition of the clathrin-dependent uptake route resulted in the loss of transfection with PEI polyplexes in several cell lines, suggesting that the clathrindependent uptake route is important for successful transfection. However, combined pathways may function at the same time. So we used several inhibitors to study cationic liposome-mediated cell uptake pathways. The results showed that chlorpromazine and wortmannin could reduce transfection efficiency by
B

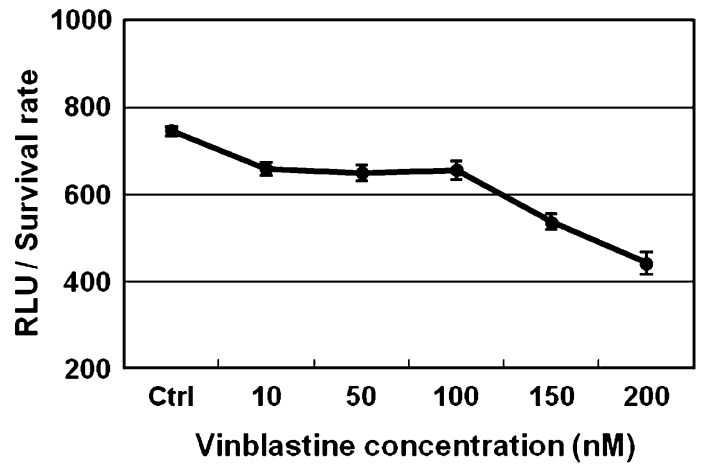

determined with the MTT method with non-treated cells as control. All results are expressed as mean $\pm \operatorname{SD}(n=4)$. b RLU/survival rate with vinblastine (10-200 nM) treated Hep2 cells

30-40 \% in terms of RLU/survival rate by inhibiting clathrin route. Chlorpromazine inhibits uptake pathway mediated via inhibiting clathrin-coated nest aggregation in the membrane. Wortmannin inhibits uptake by affecting the formation of double-shell of the endosomes to reduce the intracellular endosomal vesicles (van der Aa et al. 2007). Genistein, as inhibitor of caveolin, reduced transfection efficiency by $\sim 30 \%$. Vinblastine could reduce $40 \%$ of transfection efficiency of lipoplexes by inhibiting the function of microtubulin.

Our results indicated that transfection efficiency was decreased after incubation with inhibitors, both through the clathrin- and caveolae-mediated pathways and also through microtubulin. A correlation between clathrin-mediated or caveolae-mediated endocytosis and lipoplexes transfection was supported convincingly by several lines of evidence, including the use of 
inhibitors of endocytosis, co-localization with pathway-specific markers, and deficiencies in clathrinmediated endocytosis. Additionally, the inhibition of microtubulin probably changed the microtubule function for lipoplexes transport through microtubule polymerization by the addition of vinblastine. Cell uptake and subsequent internalization is usually related to the function of microtubule. The cytoskeleton is required for the formation and internalization of clathrin-coated pits (Yarar et al. 2005). Vinblastine can affect the actin cytoskeleton grouping into actin filament polymerization orderly and promote its dissociation. Therefore, it can inhibit the uptake pathway mediated by microtubule and microfilament.

The use of inhibitors might give rise to an unambiguous outcome because of toxic side-effects (Chittimalla et al. 2005). So we took the cell survival rate into account during the uptake study of lipoplexes into cells. The ratios of RLU to survival used in this paper, could better address the problem caused by the toxicity of inhibitors.

Taken together, these results demonstrated that the uptake of lipoplexes to Hep-2 cells was mediated via both the clathrin-mediated and caveolae-mediated endocytic pathways. Otherwise, phagocytosis mediated by microtubule-microfilament also played a role in the regulation. There is no doubt that the combined action of these endocytic pathways is essential for gene delivery, and their roles will be clarified further as the studies continue. Though we need to further elucidate the functions of inhibitors and more detailed mechanism of liposomes mediated transfection, the results of this article could provide reference for the acquisition of novel gene delivery systems.

Acknowledgments The study was supported by the National Natural Science Foundation of China (20876027 and 21176046) and the Fundamental Research Funds for the Central Universities (DC12010142 and 12010104).
Open Access This article is distributed under the terms of the Creative Commons Attribution License which permits any use, distribution, and reproduction in any medium, provided the original author(s) and the source are credited.

\section{References}

Blanchard E, Belouzard S, Goueslain L, Wakita T, Dubuisson J, Wychowski C, Rouillé Y (2006) Hepatitis C virus entry depends on clathrin-mediated endocytosis. J Virol 80:6964-6972

Chittimalla C, Zammut-Italiano L, Zuber G, Behr JP (2005) Monomolecular DNA nanoparticles for intravenous delivery of genes. J Am Chem Soc 127:11436-11441

High KA (2005) Gene therapy: the moving finger. Nature 435:577-579

Howes MT, Mayor S, Parton RG (2010) Molecules, mechanisms, and cellular roles of clathrin-independent endocytosis. Curr Opin Cell Biol 22:519-527

Mayor S, Pagano RE (2007) Pathways of clathrin-independent endocytosis. Nat Rev Mol Cell Biol 8:603-612

Obata Y, Ciofani G, Raffa V, Cuschieri A, Menciassi A, Dario P, Takeoka S (2010) Evaluation of cationic liposomes composed of an amino acid-based of an endosome. Nanomedicine 6:70-77

Payne CK, Jones SA, Chen C, Zhuang XW (2007) Internalization and trafficking of cell surface proteoglycans and proteoglycan-binding ligands. Traffic 8:389-401

Sandvig K, Pust S, Skotland T, van Deurs B (2011) Clathrinindependent endocytosis: mechanisms and function. Curr Opin Cell Biol 23:1-8

van der Aa MAEM, Huth US, Häfele SY et al (2007) Cellular uptake of cationic polymer-DNA complexes via caveolae plays a pivotal role in gene transfection in COS-7 cells. Pharm Res 24:1590-1598

von Gersdorff K, Sanders NN, Vandenbroucke R, De Smedt SC, Wagner E, Ogris M (2006) The internalization route resulting in successful gene expression depends on both cell line and polyethylenimine polyplex type. Mol Ther 14:745-753

Yarar D, Waterman-Storer CM, Schmid SL (2005) A dynamic actin cytoskeleton functions at multiple stages of clathrinmediated endocytosis. Mol Biol Cell 16:964-975

Ziello JE, Huang Y, Jovin IS (2010) Cellular endocytosis and gene delivery. Mol Med 16:222-229 